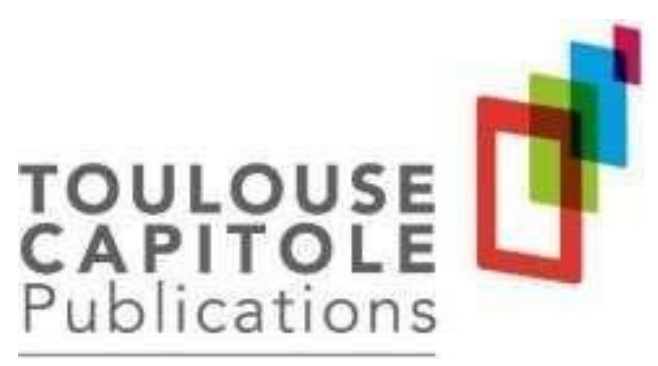

« Toulouse Capitole Publications » est l'archive institutionnelle de l'Université Toulouse 1 Capitole.

Allemagne : la lutte antiterroriste sans régime d'exception

GAILLET AURORE

Classen (C. D.)

Référence de publication: GAILlet (A.), ClASSEN (C. D.), «Allemagne : la lutte antiterroriste sans régime d'exception », Annuaire international de justice constitutionnelle, 2017.

Pour toute question sur Toulouse Capitole Publications, contacter portail- publi@ut-capitole.fr 


\section{Allemagne : la lutte antiterroriste sans régime d'exception}

$\mathrm{Si}$ « le terrorisme est un phénomène difficile à appréhender juridiquement $»^{1}$, le droit est l'un des instruments constamment sollicités pour tenter d'y apporter des réponses. Il se fonde à cet effet sur des définitions aux contours larges, mettant l'accent sur l'élément « intentionnel » des actes et « infractions » concernés comme sur leur but, tendant à « troubler gravement l'ordre public par l'intimidation », « la terreur », la « déstabilis[ation] voire la destru[ction] des structures fondamentales politiques, économiques ou sociales d'un pays $»^{2}$.

Rien de bien nouveau : la menace du terrorisme pèse sur les États européens au moins depuis les années 1970. Elle constitue depuis lors un défi pour les ordres constitutionnels, lesquels exigent de déterminer une balance adéquate entre la liberté et la sécurité. « Un tel impératif n'exclut pas seulement la poursuite d'un objectif de sécurité absolue, qui ne peut en réalité guère être atteint, sauf peut-être au prix de l'abolition totale de la liberté $»^{3}$. Conformément aux principes de la démocratie et de l'État de droit reconnus dans le monde occidental, « la volonté d'atteindre le niveau de sécurité le plus élevé possible est elle-même soumise à des restrictions juridiques, parmi lesquelles il faut surtout prendre en compte l'interdiction d'ingérences inadéquates dans les droits fondamentaux [...] $»^{4}$. Ces mots issus de la jurisprudence de la Cour constitutionnelle de Karlsruhe sont partagés bien au-delà des frontières allemandes. Reste toutefois à les mettre en œuvre.

La plupart des États européens se sont vus confrontés à des épreuves jalonnant leur propre histoire nationale, que l'on pense à la «Fraction Armée rouge » en Allemagne et aux « Brigades rouges » en Italie, motivées par des idéologies politiques, ou aux mouvements nationalistes en Espagne (Pays basque), au Royaume- Uni (Irlande du Nord) ou en France (Corse). Chaque pays a cherché en conséquence sa propre voie dans la quête de cette balance liberté/sécurité. La donne a pourtant quelque peu changé depuis les attentats du 11 septembre 2001. On constate en effet désormais une plus grande internationalisation du terrorisme. Partant, la recherche de moyens de lutte contre le terrorisme, qui seraient à la fois efficaces et les moins attentatoires possible aux principes fondamentaux de la liberté, de la démocratie et de l'État de droit invite à s'interroger plus en avant sur les solutions envisagées par d'autres pays.

Dans ce contexte, il s'agit notamment de se demander dans quelle mesure les moyens « ordinaires » de lutte contre la criminalité peuvent suffire et dans quelle mesure il faut, au contraire, recourir à des instruments d'exception comme l'a fait la France avec la déclaration de l'état d'urgence, à la suite des attentats de Paris du 13 novembre 2015. Celui-ci permet aux autorités administratives de prendre des mesures restreignant singulièrement les libertés, qu'il s'agisse d'assigner certaines personnes à résidence, de fermer certains lieux, de perquisitionner des domiciles même la nuit ou encore d'interdire les manifestations. Les nombreuses inquiétudes formulées à l'endroit desdites mesures ont été abondamment relayées en France, par les médias, la doctrine ${ }^{5}$ et la justice. Le regard comparatif n'en demeure pas moins fécond pour approfondir la question.

L'examen de la situation et des réponses allemandes trouve ici tout son intérêt. Vu de France, le regard outre-Rhin a ceci d'intéressant que, pour des raisons historiques, l'Allemagne ne connaît pas d'état d'urgence permettant de limiter les libertés de manière plus restrictive qu'en temps «normaux » (I). En outre, la législation antiterroriste existante est contrôlée de manière particulièrement dense par la Cour constitutionnelle fédérale (II). La différence d'approche peut ainsi apporter un nouvel éclairage pour les 
discussions françaises.

\section{I.- LA RÉTICENCE HISTORIQUE À L'ÉGARD DE RÉGIMES D'EXCEPTION EN ALLEMAGNE}

Ni la Constitution allemande - la Loi fondamentale du 23 mai 1949 (LF) -, ni la législation en vigueur outre-Rhin ne prévoient de régime d'état d'urgence tel qu'il existe en France. Certaines règles sont certes prévues pour régir des situations de nécessité. Mais celles-ci ne concernent que la répartition des compétences entre les organes de l'État d'une part, entre la Fédération et les Länder d'autre part (A). Comme souvent s'agissant des rapports entre liberté et sécurité, les raisons historiques demeurent essentielles à la compréhension de l'état du droit (B).

\section{A.- La difficile expérience historique de l'Allemagne en matière de régime d'exception}

La Constitution de Weimar de 1919, première constitution démocratique de l'Allemagne entrée en vigueur, avait conféré au président de la République de vastes pouvoirs exceptionnels. Son article 48 al. 2 l'autorisait notamment, « lorsque la sûreté et l'ordre publics [étaient] gravement troublés ou compromis au sein du Reich, [à] prendre les mesures nécessaires à leur rétablissement [et] en cas de besoin, [à] recourir à la force. À cette fin, il [pouvait en outre] suspendre totalement ou partiellement l'exercice des droits fondamentaux garantis aux articles 114 [liberté individuelle], 115 [inviolabilité du domicile], 117 [secret de la poste et des télécommunications], 118 [liberté d'opinion], 123 [liberté de réunion et de manifestation], 124 [liberté d'association] et 153 [droit de propriété] de la Constitution ». Le Parlement (Reichstag) n'avait qu'un rôle limité, dès lors qu'il se voyait simplement « communiquer sans délai les mesures prises » (art. 48 al. 3).

Le large pouvoir discrétionnaire ainsi accordé au Président, correspondant à l'interprétation dominante de la Constitution ${ }^{6}$, n'a pas manqué de faire l'objet d'un usage répété dans le contexte troublé de la jeune et fragile République de Weimar. L'article 48 a ainsi été appliqué à maintes reprises dès les premières années de la République (1919-1925). Il a ensuite surtout été appliqué de manière quasi permanente au cours des années 1930 à 1933, années particulièrement difficiles marquées, sur le plan politique, par l'absence de soutien parlementaire au gouvernement ( gouvernements présidentiels ${ }^{7}$ ). C'est ainsi qu'en 1931, le vote par le Parlement de quelque trente et une lois n'a pas empêché le Président d'adopter quarante-quatre ordonnances de nécessité sur le fondement de l'article 48 al. 2. Cette politique a bénéficié du soutien au moins passif du grand parti d'opposition qu'était alors le parti social-démocrate. Celui-ci n'a en effet jamais essayé de faire abroger par le Reichstag les mesures prises, possibilité pourtant envisagée à l'article 48 al. 3. C'est ainsi que l'exception s'est progressivement ancrée dans la vie juridique "normale ", l'urgence et les pouvoirs exceptionnels devenant la règle.

Sans doute l'ordonnance du 28 février $1933^{8}$ mettant hors de vigueur de nombreux droits fondamentaux après l'incendie du Reichstag le 5 février sera-t-elle retenue par l'histoire comme ayant franchi le pas décisif vers la dictature nazie. Elle s'inscrit cependant dans la lignée de la transgression du normal par l'exceptionnel, problématique toujours au cœur des débats contemporains - notamment français. En Allemagne, au contraire, la Loi fondamentale s'est efforcée de prévenir de tels glissements.

B.- Les états de nécessité sous la Loi fondamentale de 1949 


\section{1.- La délicate question de la précision de l'état de nécessité après la Seconde Guerre mondiale}

Chaque constitution est le fruit de l'histoire, cherchant notamment à ne pas reproduire les échecs et difficultés de celles qui l'ont précédée. Rien n'est plus vrai pour la Loi fondamentale, placée face au défi de la rénovation de la démocratie et de l'État de droit après la douloureuse page du national-socialisme. La place première du catalogue de droits fondamentaux, définis comme liant les trois pouvoirs, y compris donc le législateur « à titre de droit directement applicable » (art. 1 al. 3 LF) est à comprendre dans cette perspective, de même que le rôle essentiel de la Cour constitutionnelle fédérale. Le système parlementaire rénové prévoit en outre une fonction présidentielle essentiellement représentative et symbolique, le Président étant du reste désormais élu par une Assemblée spéciale (Bundesversammlung) et non plus directement par le peuple. Une telle configuration ne peut évidemment pas s'accorder avec des pouvoirs exceptionnels forts. Le rejet de l'inscription de tels pouvoirs dans la Loi fondamentale, au profit de quelque organe que ce soit, a fait l'objet d'un large consensus ${ }^{9}$.

Pareil état du droit n'a guère posé de problème en 1949, au moment de l'entrée en vigueur de la Constitution allemande. L'Allemagne était en effet encore sous la tutelle des Alliés, vainqueurs de la Seconde Guerre mondiale. Ceux-ci s'étaient vus conférés de larges pouvoirs sur l'Allemagne, y compris de nécessité. Lorsque, en 1955, l'Allemagne retrouve sa souveraineté, les puissances alliées ne sont en outre pas immédiatement dessaisies de l'ensemble de leurs pouvoirs en la matière. Il est prévu à ce titre que « les droits des trois puissances antérieurement détenus ou exercés par elles en ce qui concerne la protection de la sécurité des forces armées stationnées sur le territoire de la République fédérale, et qui sont temporairement conservés, disparaîtront lorsque les autorités allemandes compétentes auront obtenu des pouvoirs similaires, en vertu de la législation allemande, leur permettant de prendre des mesures effectives pour protéger la sécurité de ces forces, y compris la possibilité de faire face à une atteinte grave portée à la sécurité et à l'ordre publics $[\ldots] »^{10}$.

Les tentatives ultérieures d'adopter les lois nécessaires s'analysent aisément comme contribuant à l'effort allemand de s'affirmer comme une puissance indépendante, tendant également à rendre caducs les pouvoirs des puissances alliées ${ }^{11}$. Il faudra pourtant attendre 1968 pour que des « lois relatives à l'état de nécessité » (Notstandsgesetze) trouvent leur place dans la Constitution. La révision constitutionnelle se fera à la faveur d'un consensus au sein de la première « grande coalition », les deux grands partis allemands (l'Union démocratique chrétienne et la social-démocratie - CDU et SPD) disposant alors de plus de deux tiers des voix au Parlement.

Outre les divers régimes régissant l'état de nécessité qui seront abordés par la suite, la révision de 1968 a enrichi la Constitution dans deux directions. D'une part, il faut noter un pas dans le sens d'une restriction des droits fondamentaux dès lors que, dans certains cas, en matière de secret de la correspondance, de la poste et des télécommunications, l'autorisation judiciaire des écoutes téléphoniques par les autorités publiques a été remplacée par la seule autorisation d'une commission parlementaire ${ }^{12}$. D'autre part, d'autres mesures ont visé à contrebalancer cet assouplissement des contre-pouvoirs. C'est ainsi que 1'article 20 al. 4 reconnaît désormais le droit de résistance à l'oppression. C'est aussi à cette occasion que le recours constitutionnel individuel a été consacré au niveau constitutionnel (art. 93 al. $1 \mathrm{n}^{\circ} 4 \mathrm{aLF}$ ) - auparavant simplement prévu par la Loi fédérale sur la Cour constitutionnelle de 1951.

En dépit de cette recherche d'équilibre, le débat sur les lois de nécessité a rencontré une vive opposition 
auprès de larges franges de l'opinion publique, relayée par les syndicats, les partis politiques « minoritaires » et la jeunesse, dans le contexte tendu de 1968. Une fois encore, l'expérience historique a été mobilisée afin de mettre en avant les risques encourus pour la démocratie et l'État de droit ${ }^{13}$. Le droit aujourd'hui en vigueur n'en est pas moins directement issu de cette révision.

\section{2.- Les régimes actuels des états de nécessité}

Depuis 1968, la Loi fondamentale contient ainsi divers régimes d'états de nécessité.

Outre l'état de nécessité « législatif» (art. 81 LF) - qui concerne l'adoption d'un projet de loi dans une situation de blocage politique -, on peut distinguer des règles spécifiques régissant un état de nécessité intérieur et un état de nécessité extérieur. Le premier vise à « maintenir ou restaurer la sécurité ou l'ordre publics [...] : dans des « cas particulièrement importants », l'article 35 al. 2 LF autorise un Land à faire appel aux forces fédérales pour l'assister. Dans le même sens, l'article 91 al. 1 LF prévoit le soutien des forces de police et des frontières des Länder entre eux ou l'appel aux forces fédérales pour écarter " un danger menaçant l'existence ou l'ordre constitutionnel libéral et démocratique de la Fédération ou d'un Land ». Un pas supplémentaire peut être franchi avec l'appel aux forces armées (art. 87a al. 4 LF). La lecture de ces dispositions met en avant l'importance centrale de la question de la répartition des compétences dans le cadre du fédéralisme allemand.

S'agissant de l'état de nécessité extérieur, supposant que le territoire fédéral fasse l'objet d'une attaque armée (art. 115 LF) ou que des tensions internationales laissent envisager une telle attaque (art. 80 a LF) ${ }^{14}$, le constat d'une telle situation par le Bundestag conduit également à des aménagements de la répartition du pouvoir législatif entre la Fédération et les Länder - la concentration renforcée des pouvoirs modifiant le plus souvent les équilibres fédératifs au détriment des Länder - et à la mise en place d'une procédure législative spécifique (art. 115a s. LF). Sont en outre prévus de classiques transferts de compétences entre autorités civiles et militaires (art. 87a al. 3 LF).

Des garde-fous sont par ailleurs prévus, emportant notamment l'interdiction de dissoudre le Bundestag (art. 115h LF) et de « porter atteinte au statut et à l'exercice des missions constitutionnelles de la Cour constitutionnelle fédérale et de ses juges » (art. 115g LF). L'importance de ces deux organes constitutionnels dans la démocratie parlementaire allemande est ce faisant confirmée, y compris donc en cas d'état de nécessité. De même, les droits fondamentaux demeurent pour l'essentiel préservés ${ }^{15}$. Sans doute certains transferts de compétences des autorités civiles aux autorités militaires emportent-ils un risque d'ingérence accrue. Mais les pouvoirs de l'État envers les citoyens n'en demeurent pas moins inchangés et les droits fondamentaux garantis par la Loi fondamentale sous la protection de la Cour constitutionnelle fédérale n'ont nulle vocation à être suspendus comme ce fut le cas sous Weimar.

S'agissant plus spécifiquement de la lutte contre le terrorisme, une question épineuse tient à la possibilité de qualifier une attaque terroriste d'attaque armée contre le territoire, permettant ainsi d'actionner l'un des états de nécessité. Il est généralement admis que ladite attaque doit être de nature à porter atteinte au bon fonctionnement des institutions de l'État. Or, si le risque direct est bien avéré en cas d'attaque par une armée étrangère régulière, il est plus difficile à identifier en cas d'attaque terroriste plus diffuse ${ }^{16}$.

Le cadre constitutionnel de la lutte contre le terrorisme demeure dès lors à préciser. À cet effet, il faut se tourner vers la jurisprudence pointilleuse de la Cour constitutionnelle, telle que développée dans le cadre 
du contrôle de nombreuses lois récentes.

\section{II.- LE CADRE CONSTITUTIONNEL ACTUEL DE LA LUTTE ANTITERRORISTE}

\section{A.- Les mesures adoptées dans les années 1970}

L'inexistence d'un régime spécial en matière de lutte antiterroriste ne signifie pas que l'Allemagne soit désarmée dans la lutte contre le terrorisme. La possibilité d'écoutes téléphoniques sans recours juridictionnel a déjà été mentionnée. Cet assouplissement a été autorisé par la Cour constitutionnelle sous certaines réserves, renforçant notamment les possibilités d'information de l'intéressé ${ }^{17}$.

Les difficiles années soixante-dix marquées par les attentats perpétrés par la Fraction armée rouge ont également conduit à préciser certaines mesures. C'est ainsi qu'en 1977, alors que plusieurs attentats terroristes graves secouaient la République Fédérale, le législateur a prévu la possibilité d'interdire à certains prisonniers, pour une durée limitée, tout contact avec toute personne extérieure, y compris avocats. Cette possibilité est depuis lors prévue dans le droit commun ${ }^{18}$.

\section{B.- L'utilisation des forces armées}

En Allemagne comme ailleurs, l'attentat du 11 septembre 2001 a soulevé des discussions sur la capacité des forces de l'ordre à faire face à une situation identique. En réaction, le législateur de 2005 a adopté une loi permettant au gouvernement d'ordonner une intervention armée contre des avions en cas de détournement. Pareille possibilité - impliquant, le cas échéant, la destruction de l'avion concerné - a été prévue au seul cas où ce dernier devait être utilisé contre des vies humaines et si aucun autre moyen alternatif n'était possible ${ }^{19}$.

Quels que soient les garde-fous envisagés, la loi n'a pas manqué de soulever de nombreux recours constitutionnels, individuels et abstraits ${ }^{20}$. La réponse de la Cour constitutionnelle allemande les a confortés, considérant la possibilité d'abattre un avion comme une atteinte non seulement au droit à la vie, mais également à la dignité humaine. Or, selon la Constitution, cette dernière est «inviolable $»^{21}$ et ne peut faire l'objet d'aucune révision constitutionnelle (art. 79 al. 3 LF). Pareil recours à la dignité humaine a été à son tour fortement critiqué par la doctrine. Celle-ci a en effet rappelé qu'en cas de guerre, certaines mesures peuvent s'avérer indispensables à la préservation de la vie elle-même et qu'il est alors difficile de distinguer, comme le raisonnement de la Cour semblait y inviter, deux vies et dignités différentes ${ }^{22}$. On perçoit ici l'intensité des débats suscités.

Par-delà cette question, le climat récent de menaces à la sécurité et la volonté d'y faire face ont conduit l'Allemagne à renforcer son arsenal législatif, prévoyant notamment des mesures de surveillance des personnes et d'utilisation des informations collectées. Si ce nouvel activisme législatif rapproche ce faisant l'Allemagne de ses voisins européens, la Cour constitutionnelle s'est efforcée de l'encadrer. Les saisines successives l'ont ainsi conduit à rendre une douzaine de décisions à ce sujet ${ }^{23}$. Ces lois et décisions les encadrant constituent dès lors le cadre actuel de la lutte antiterroriste en Allemagne.

\section{C.- Les mesures de surveillance et leur encadrement jurisprudentiel}

\section{1.- Aperçu global de la jurisprudence constitutionnelle}


La plupart des affaires ${ }^{24}$ ont ceci de commun qu'elles ont été rendues à la suite d'un recours constitutionnel individuel, mais dans le but réel de susciter un contrôle abstrait de la loi visée. Dans ce cadre, toutes les lois concernées ont fait l'objet d'une annulation partielle, deux d'entre elles ont même fait l'objet d'une censure totale. Les deux décisions les plus récentes révèlent de surcroît la grande difficulté à trouver une solution acceptable pour l'ensemble des juges de la Première Chambre de la Cour, compétente en la matière. C'est ainsi que la décision du 24 avril 2013 examinant une loi créant un fichier antiterrorisme (Antiterrordatei) n'a conclu à la constitutionnalité partielle de la loi qu'à la faveur d'un partage des voix ${ }^{25}$. Dans le même ordre d'idée, la décision du 20 avril 2016 examinant la loi sur l'Office fédéral de lutte contre la criminalité a opté pour une inconstitutionnalité partielle, mais présentant un vote partagé, à 5 voix contre 3, accompagné de deux opinions séparées. Les divisions témoignent ici encore de l'extrême difficulté de la recherche d'un équilibre entre la liberté et la sécurité.

En toute hypothèse, il ne s'agit pas de présenter le cadre constitutionnel allemand de la lutte contre le terrorisme de manière exhaustive, mais bien plus d'en donner une perspective d'ensemble. Il convient du reste de garder à l'esprit le fait que certaines lois en vigueur n'ont pas - encore ? - fait l'objet d'un contrôle constitutionnel. Tel est le cas de la deuxième loi sur la conservation de données de télécommunication, adoptée en 2016. En outre, l'aperçu ici proposé a sans doute vocation à n'être que provisoire, au regard des propositions qui ne manquent pas d'être défendues à chaque nouvelle menace - la vidéosurveillance des places publiques fait ainsi partie des mesures souvent discutées. Surtout, une nouvelle loi sur l'Office fédéral de police criminelle (Bundeskriminalamt - BKA) vient d'être adoptée et il faudra suivre de près les possibles aménagements du fédéralisme allemand en la matière ${ }^{26}$.

Pour l'heure, il est possible de revenir sur certains éléments des législations et mesures « antiterroristes », telles qu'elles ont été entérinées et orientées par la Cour de Karlsruhe.

\section{2.- Les droits fondamentaux concernés}

Les mesures de surveillance constituent avant tout des ingérences dans les garanties de la vie privée prévues par la Loi fondamentale. Il s'agit notamment du secret des télécommunications (art. $10 \mathrm{LF})^{27}$, de la liberté du domicile (art. 13 LF) ${ }^{28}$ en tant que garanties spéciales, ainsi que du « droit général de la personnalité », développé par la jurisprudence constitutionnelle à partir des années $1950^{29}$ sur le fondement de la liberté générale (art. 2 al. 1 LF) et de la dignité humaine (art. 1 al. 1 LF) ${ }^{30}$. La définition ouverte de ces derniers droits a permis à la Cour d'adapter la protection constitutionnelle à des développements techniques modernes, non envisagés initialement, et à faire ainsi du catalogue des droits fondamentaux de la Loi fondamentale un living instrument ${ }^{31}$. Partant, celui-ci inclut désormais le droit à la protection des données ${ }^{32}$ ou encore le principe de l'inviolabilité des systèmes techniques d'information (Unverletzlichkeit der informationstechnischen Système) ${ }^{33}$. De plus, la Cour n'a de cesse de souligner que la dignité humaine protège un noyau inviolable du droit de la personnalité, ce qui lui confère un caractère intangible ${ }^{34}$. Précisons dès ici que ces droits ne limitent pas la seule collecte d'informations : l'utilisation de ces informations de même que leur transfert à une autre autorité sont également considérés comme des ingérences ${ }^{35}$. En revanche, les droits tels que la liberté d'opinion et de réunion n'ont pratiquement jamais été touchés par les réglementations. Quant à la liberté de la presse et de la radio (art. 5 al. 1 phrase $2 \mathrm{LF}$ ), elle n'a joué un rôle que dans une affaire concernant le contrôle de données de télécommunication (Verbindungsdaten) d'une station de télévision ${ }^{36}$. 


\section{3.- Les conditions formelles encadrant les ingérences}

La constitutionnalité des lois successivement adoptées aux fins de lutte contre le terrorisme a été soumise à différentes conditions formelles. Comme souvent, la forme n'est jamais qu'une seule question de formalité ${ }^{37}$, et cela vaut tout particulièrement lorsqu'il s'agit de déplacer le curseur de l'équilibre entre liberté et sécurité.

Il appartient en premier lieu au législateur d'agir dans le cadre de ses compétences. Cela n'a guère posé de problème, la Cour n'ayant jamais été amenée à considérer qu'une compétence de la Fédération manquait de base légale. Plus en avant, à l'instar de tous les cas d'ingérence dans les droits fondamentaux, et plus encore lorsqu'est en cause un aspect de la liberté personnelle, la Cour est particulièrement attentive à l'étendue des précisions apportées par le législateur. Celui-ci doit ainsi définir clairement, pour chaque domaine, les mesures autorisées, les menaces concernées, les délits (supposés) et le degré de soupçon à même d'emporter leur caractérisation. Il lui revient également de préciser les autorités compétentes ainsi que, in fine, le cadre et l'objectif de l'utilisation des informations collectées ${ }^{38}$. Dans les cas où l'administration peut concrétiser les conditions permettant l'utilisation de certaines données, ces conditions doivent être publiées ${ }^{39}$. La transparence du niveau de protection de données vise ce faisant à maintenir la confiance des citoyens et à garantir une certaine sécurité juridique ${ }^{40}$.

Au-delà, la Cour contraint le législateur à prévoir des procédures de protection adéquates en cas d'atteintes graves ${ }^{41}$. L'État doit notamment s'efforcer de collecter les informations de la manière la plus ouverte possible $^{42}$. Une collecte secrète n'est permise que si cela est rendu nécessaire par l'inefficacité d'une collecte ouverte $^{43}$. De même, l'intéressé doit être informé au plus tôt ${ }^{44}$. En principe, il a ainsi le droit de connaître les informations détenues par l'État ${ }^{45}$. Une exception existe pour les personnes surveillées de manière aléatoire, dont les informations ont été effacées, et qui n'ont de ce fait pas d'intérêt spécifique à être informées - en poussant trop loin l'analyse, une telle information pourrait même constituer une nouvelle ingérence ${ }^{46}$.

Ces mesures d'information sont complétées par d'indispensables précisions relatives au rôle du juge, même si, à la différence de la France, la Loi fondamentale ne le qualifie pas expressément de " gardien de la liberté individuelle ». Dans certains cas, l'intervention du juge doit être préalable aux mesures de surveillance. La Constitution le prévoit elle-même en matière de garantie du domicile (art. 13 al. 2 à 6 LF)

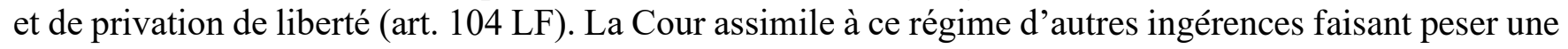
menace particulièrement grave sur la liberté individuelle : tel est par exemple le cas des perquisitions des systèmes d'information ${ }^{47}$, de la conservation des données de télécommunication ${ }^{48}$ ou encore de la détention d'informations sur une durée plus longue ${ }^{49}$. Dans tous ces cas, il revient au juge d'examiner de près la mesure et de motiver de manière précise sa décision ${ }^{50}$. S'agissant des systèmes d'information, souvent concernés en matière de mesures antiterroristes, une difficulté supplémentaire tient à l'impossibilité de déterminer avec précision et par avance les données échangées : dans ce cas, l'évaluation et le contrôle sont transférés à des autorités indépendantes, travaillant en lien étroit avec les services compétents ${ }^{51}$.

Enfin, un régime de surveillance efficace et conforme à l'État de droit allemand suppose un certain cadre institutionnel. Cela exige des protocoles systématiques en matière d'activités de surveillance ${ }^{52}$. Lorsque les données collectées de manière illégale ou inutilisable car violant le noyau dur du droit de la personnalité doivent être effacées rapidement ${ }^{53}$, la célérité ne doit pas pour autant emporter une 
précipitation préjudiciable qui rendrait impossible tout contrôle ${ }^{54}$. Le cadre institutionnel est ici renforcé par le rôle du Commissaire chargé de la protection des données, autorité indépendante dotée de pouvoirs importants ${ }^{55}$, et par la présentation d'un rapport régulier au Parlement ${ }^{56}$.

\section{4.- Les conditions matérielles : objectifs légitimes et proportionnalité}

Sur le fond, la Cour constitutionnelle a également précisé les conditions aptes à encadrer les mesures prises dans le cadre de la lutte contre le terrorisme.

Il s'agit en premier lieu des exigences de proportionnalité des mesures, principe fondamental en droit allemand $^{57}$. De manière générale, il suppose que les objectifs soient légitimes et que les mesures prises soient adéquates, nécessaires et proportionnées pour justifier les ingérences dans les droits fondamentaux ${ }^{58}$. L'adéquation et la nécessité n'ont jamais posé de problème spécifique dans le cadre de la lutte contre le terrorisme. À l'inverse, la Cour veille, d'une part à ce que les mesures de surveillance soient justifiées par des soupçons suffisamment graves et concrets, d'autre part à une protection suffisamment efficace du noyau dur de la vie privée ${ }^{59}$.

En outre, les évaluations et perspectives sur lesquelles les autorités fondent leur action doivent être basées sur des faits concrets (tatsachengestützt) et non sur une seule appréciation générale (erfahrungsgestützt) ${ }^{60}$. Même les intérêts les plus importants ne doivent pas conduire à écarter cette condition de probabilité suffisante de réalisation de la menace ${ }^{61}$. Dans le même sens, les personnes visées par des mesures de surveillance doivent avoir un lien particulier avec une activité de terrorisme ${ }^{62}$.

En matière de surveillance du domicile et des systèmes d'information, il est certes inévitable que des personnes tierces soient visées. Mais cela doit se limiter à ce qui est strictement nécessaire dans le cadre de la surveillance ${ }^{63}$. Au-delà, des délits de moindre importance ne permettent pas une ingérence dans des droits aussi sensibles que la liberté de la communication, du domicile et de la liberté des systèmes d'information ${ }^{64}$.

En matière de droits et libertés, tout est affaire de conciliation et de pondération. Le droit allemand apporte cependant une spécificité, considérant que la dignité humaine ne peut pas faire l'objet d'une telle pondération, par quelque objectif que ce soit. Cela est d'autant plus délicat que, on l'a dit, la dignité inclut notamment le noyau dur de la vie privée (Kernbereich privater Lebensführung) ${ }^{65}$. Dans ce cadre, il revient à l'État de prévoir des règles prévenant de telles ingérences. S'il devait apparaître que l'État se livrait malgré tout à des opérations de collectes d'information, la mesure en question devrait être immédiatement interrompue, les informations obtenues effacées et interdites de toute utilisation ultérieure ${ }^{66}$. Cela vaut tant au niveau de la collecte que de l'utilisation des données ${ }^{67}$.

Le «noyau de la personnalité » ainsi protégé emporte de surcroît l'interdiction d'une « surveillance totale

»(Rundumüberwachung) dans le temps et l'espace, qui conduirait inévitablement à atteindre ledit noyau ${ }^{68}$. L'État n'a en outre pas le droit d'exercer une surveillance secrète complète, en créant par exemple un profil et suivi complet des mouvements d'une personne ${ }^{69}$. En cas de mesures successives et/ou cumulatives, s'ajoute également l'encadrement du cumul de ces mesures ${ }^{70}$.

S'agissant enfin de la situation de certaines professions spécifiques, la Cour se montre particulièrement vigilante. Aussi rappelle-t-elle que la communication établie avec certaines personnes - ministres d'un 
culte, avocats, médecins - risque de toucher au noyau dur de la vie privée ${ }^{71}$. Pour les journalistes, elle se fonde sur l'article 5 al. $1^{\text {er }}$ phrase 2 LF, disposition spéciale applicable (liberté de la presse et de la radio) ${ }^{72}$.

\section{5.- L'échange de données entre services}

La recherche d'une pondération adéquate - et donc constitutionnelle - a enfin des incidences en matière de répartition des compétences entre la police et les services de renseignement. La Cour de Karlsruhe a ainsi rappelé que, si les services de renseignement disposent de larges pouvoirs pour collecter des informations, ils ne bénéficient guère de compétences opérationnelles ${ }^{73}$. À l'inverse, si la police dispose certainement de ces dernières, son action suppose des faits concrets ${ }^{74}$ et doit être la plus ouverte possible ${ }^{75}$. Est ainsi évoqué un « principe de séparation de pouvoirs informationnels 》 (informationelles Trennungsprinzip $)^{76}$. Une telle distinction est essentielle dès lors qu'elle emporte des conséquences en matière d'encadrement de l'utilisation des données ${ }^{77}$. L'intégration de données provenant de diverses sources dans un même fichier doit garantir que les principes mentionnés ci-dessus sont respectés ${ }^{78}$. Tout contournement de limites de compétence d'une autorité par une autre est prohibé ${ }^{79}$.

En outre, lorsque des données sont stockées, il convient de préciser celles qui ont été obtenues à la faveur d'une ingérence dans la liberté de la communication, du domicile ou de la liberté des systèmes d'information ${ }^{80}$. Il s'agit de garantir que ces données ne seront utilisées que si les conditions de leur collecte sont respectées. Le cas échéant, l'utilisation sera jugée inconstitutionnelle ${ }^{81}$. De même, la transmission de ces données à d'autres autorités doit être entourée de garanties suffisantes ${ }^{82}$.

En ce qui concerne enfin la coopération avec des services étrangers, la Cour ne s'y oppose pas par principe. Mais elle n'a de cesse de rappeler que, si la protection assurée ailleurs peut être différente, le respect de ces différences ne doit pas conduire à contourner les limites imposées par le droit allemand. Les données dont la collecte a été jugée incompatible avec les droits fondamentaux ne peuvent par ailleurs pas être utilisées ${ }^{83}$. Conformément aux principes sans cesse rappelés, la Cour met en avant la nécessité d'objectifs revêtus d'une importance suffisante, d'un standard suffisant de garanties dans l'État récepteur et, sur le plan intérieur, de critères légaux clairs, d'un système indépendant de contrôle en matière de protection de données et d'un contrôle juridictionnel efficace ${ }^{84}$. Ce sont autant de critères tendant à établir un certain niveau de confiance réciproque, à la base même de l'échange et de la coopération.

\section{D.- Spécificités du contrôle constitutionnel}

S'agissant enfin des possibilités procédurales permettant aux citoyens de contester les mesures dont ils peuvent faire l'objet dans le cadre de la lutte contre le terrorisme, le recours constitutionnel individuel confirme sa place centrale en droit allemand. Sans doute les conditions qui l'entourent exigent-elles que le demandeur fasse valoir une lésion personnelle et directe dans l'un de ses droits fondamentaux. Toutefois, selon une jurisprudence constante, la Cour constitutionnelle accepte par exemple les recours lorsque le requérant n'a pas été informé de la mesure le concernant - ce qui, dans le cadre de la lutte antiterroriste, n'est pas qu'un cas d'école ${ }^{85}$.

Pour certaines lois ici en question, leur inconstitutionnalité a fréquemment emporté leur annulation pure et simple ${ }^{86}$. Dans d'autres cas, la Cour s'est néanmoins contentée de déclarer l'inconstitutionnalité et de prescrire au législateur d'adopter de nouvelles règles dans un certain délai. Cette modulation tient, comme bien souvent en la matière, aux risques qu'une nullité immédiate pourrait causer aux objectifs d'intérêt 
général dotés d'une grande importance - lesquels, en l'espèce, peuvent primer, du moins un temps, sur les droits fondamentaux ${ }^{87}$.

\section{Conclusion}

Pour des raisons historiques l'Allemagne ne dispose pas d'un arsenal spécifique de mesures d'urgence. Il en va de même pour la lutte antiterroriste. Toute mesure doit donc répondre aux exigences constitutionnelles ordinaires. La Cour constitutionnelle allemande s'attache en conséquence à examiner les dispositions qui lui sont soumises, ce qu'elle fait avec une intensité remarquable. Elle exige en particulier du législateur - qui n'échappe pas à la tentation de l'inflation législative qui caractérise d'autres pays européens - une grande précision dans la détermination des conditions des ingérences, notamment lorsqu'il s'agit de mesures de surveillance. Elle vérifie en outre que seuls des soupçons d'une importance suffisante justifient ces mesures, tout en veillant à une protection efficace du noyau dur de la vie privée.

$\mathrm{Au}$ total, la jurisprudence constitutionnelle allemande révèle, une fois encore, la spécificité de son contrôle. L' « omnipuissance » et l' « omniprésence » ${ }^{88}$ qui la caractérisent sont assez largement acceptées par la doctrine ${ }^{89}$, les critiques fondamentales étant plutôt rares ${ }^{90}$. On peut toutefois douter qu'un tel pouvoir du juge constitutionnel soit accepté au-delà des frontières allemandes.

\section{Notes de bas de page}

P. CHAUDON, in J. L. Gillet, P. Chaudon, W. Mastor, « Terrorisme et liberté », Constitutions 2012, p. 403 sq.

2 Pour des définitions concrètes v. art. 2 al. 2 de la directive 2017/541 de 1'Union européenne ; art. 421-1 du Code pénal français ; $§ 129$ a du Code pénal allemand. BVerfGE 115, 320 (358).

Ibid.

Voir notamment O. BEAUD, C. GUÉRIN-BARGUES, L'état d'urgence. Étude constitutionnelle, historique et critique, Paris, LGDJ, 2016.

6 G. ANSCHÜTZ, Die Verfassung des Deutschen Reiches, 14e éd., Berlin, Georg Stilke, 1933, p. 278 sq. V. aussi C. GUSY, Weimar - die wehrlose Republik?, Tübingen, Mohr Siebeck, 1991, p. 53 sq.

7 C. GUSY, Die Weimarer Reichsverfassung, Tübingen, Mohr Siebeck, 1997, p. $403 s q$; selon lui la Constitution est devenu caduque (« leerlaufende Verfassung »).

8 Verordnung des Reichspräsidenten zum Schutz von Volk und Staat vom 28. Februar 1933, Reichsgesetzblatt (Journal Officiel du Reich) 1933, p. 83.

9 H. HOFMANN, Die Entwicklung des Grundgesetzes von 1949 bis 1990, in J. Isensee/P. Kirchhof (dir.), Handbuch des Staatsrechts, Heidelberg, C.F. Müller, vol. 1, $3^{\mathrm{e}}$ éd. 2003, § 9, pt. 53.

$10 \quad$ Art. 5 al. 2 de la Convention modifiée sur les relations entre les Trois Puissances et la République fédérale d'Allemagne, signée à Bonn le 26 mai 1952, non ratifiée par la France, mais reprise et modifiée par les Accords de Paris, conclus par la Conférence des Quatre (23 octobre 1954), JO allemand 1954 II p. 216 $s q$. Stipulation qui se poursuit ainsi : «Dans la mesure où ces droits continuent à pouvoir être exercés, ils ne seront exercés qu'après consultation du Gouvernement fédéral, pour autant que la situation militaire n'exclura pas une telle consultation, et si le Gouvernement fédéral reconnaît que les circonstances requièrent que ces droits soient exercés. Sous tous ses autres aspects, la protection de la sécurité de ces 
forces sera régie par les dispositions de la Convention sur les Forces ou par les dispositions de l'accord qui la remplace et, sauf dispositions contraires figurant dans tout accord applicable, par le droit allemand $»$.

29 BGHZ 13, 334 (338); BVerfGE 27, 1 (6).

30 BVerfGE 112, 304 ; 115, 320 (341 sq.) ; 120, 274 (346 sq.).

31 CEDH, v. par ex. arrêt du 25.4.1978, 5856/72 (Tyrer), § 31.

32 Depuis BVerfGE 65, 1 (41 sq.).

33 BVerfGE 120, 274 (302 sq.). 
Infra note 65.

35 BVerfGE 61, 1 (43).

36 BVerfGE 107, 299 (329 sq.).

37 Voir notamment pour cette question : O. JOUANJAN, Justifier l'injustifiable. L'ordre du discours nazi, Paris, Puf, Léviathan, 2017.

38 BVerfGE 100, 313 (372) ; 109, 279 (318, 347 sq.) ; 110, 33 (52 sq., 70 sq.) ; 113, 348 (376 sq.) ; 120, 274 (315 sq., 342) ; 125, 260 (333) ; 133, 277 pt. $140 s q$.

39 BVerfGE 133, 277 pt. 183 sq.

40 BVerfGE 125, 260 (325 sq.); 133, 277 pts. 205 sq.

41 BVerfGE 109, 279 (334)

42 BVerfGE 125, 260 (336).

43 BVerfGE 49, 329 (342 sq.) ; 100, 13 (361).

44 BVerfGE 109, 279 (364 sq.); 125, 260 (336); 129, 208 (251) ; 141, 220 pt. 136.

45 BVerfGE 141, 220 pt. 137.

46 BVerfGE 125, $260(337) ; 129,208$ (251).

47 BVerfGE 120, 274 (331).

48 BVerfGE 125, $260(337 s q)$.

49 BVerfGE 141, 220 pt. 174.

50 BVerfGE 109, 279 (358 sq.) pour la surveillance du domicile, 120, 274 (152) pour la perquisition d'un ordinateur, 125, 260 (338) pour l'utilisation d'informations obtenues par la conservation des données de télécommunication ; 141, 220 pt. 235 sur l'obligation de motivation des décisions.

51 BVerfGE 141, 220 pts. 218, 224.

52 BVerfGE 133, 277 pt 215 ; BVerfGE 141, 220 pt. 141.

53 BVerfGE 109, 279 (332 s.) ; 113, $348(392) ; 120,274$ (337, 339).

54 BVerfGE 141, 220 pts. 129, 226, 246 et 272.

55 BVerfGE 141, 220 pts. 216 et 266 sq.

56 BVerfGE 141, 220 pt. 268.

57 M. JESTAEDT, O. LEPSIUS, Verhältnismäßigkeit: Zur Tragfähigkeit eines verfassungsrechtlichen Schlüsselkonzepts (Recht - Wissenschaft - Theorie), Tübingen, Mohr, 2015.

58 C. HILLGRUBER, § 201 ( «Grundrechtsschranken »), in J. Isensee/P. Kirchhof, op. cit., vol. 9, 2011, pt. 51 sq. ; A. GAILLET, «La grenouille française et le boeuf allemand », in Ph. COSSALTER/C. WITZ (dir.), Soixante ans d'influences juridiques réciproques franco-allemandes : Jubilé des 60 ans du Centre juridique franco-allemand, Université de la Sarre, Paris, Société de Législation Comparée, coll. Droit comparé et européen, 2016, p. 163 (176 sq.).

59 Pour une collecte mettant en cause la confidentialité : BVerfGE 107, 299 (321); 113, 348 (383). 120, 274 (323 sq.). Dans ces cas, la collecte d'information n'est permise que si la vie, le corps ou la liberté des personnes, l'existence et la sécurité de l'État sont en danger ou encore s'il s'agit de lutter contre un danger commun. Par contre une telle collecte est interdite s'il s'agit d'assurer une protection d'autres droits ou intérêts : BVerfGE 125, 260 (330) ; 141, 220 pt 108.

$60 \quad$ BVerfGE 110,33 (61) ; 113, 348 (377 sq.) ; 125, 260 (328) ; 141, 220 pt. 164, 213.

61 BVerfGE 120, 274 (327).

62 Voir par exemple : BVerfG 133, 277 pt. 149 sq. ; 141, 220 pt. 150 ; BVerfGE 141, 220 pts. 165, 232.

63 BVerfGE 109, 279 (353 sq.) ; 141, 220 pt. 115.

64 BVerfGE 100, 313 (385) ; 109, 279 (348 sq.) ; 113, 348 (388 sq.) ; 120, 274 (334), 125, 260 (352 sq.), 141, 220 pt. 316. 
65 BVerfGE 109, $279(310 s q) ; 141,220$ pt. 120.

66 BVerfGE 109, 279 (332 sq.) ; 141, 220 pt. 120 sq. et 197b sq. Pour la surveillance du domicile, 113, 348 (392 sq.) et 129, 208 (245sq.) pour celle de la télécommunication électronique, 120, 274 (336 sq.) pour la perquisition d'ordinateurs et ibid., 343, pour d'autres recherches sur internet ; 141, 220 pt. 176 pour d'autres situations de la vie privée.

67 BVerfGE 141, 220 pt. 204.

68 BVerfGE 109, $279(323) ; 112,304$ (319).

69 BVerfGE 130, 1 (24); 141, 220 pts. $130 s q$.

70 BVerfGE 141, 220 pt. 130.

71 BVerfGE 109, $279(321)$; 129, 208 (247) ; s'agissant des avocats v. en outre 141, 220 pt. 257.

72 BVerfGE 107, 299 (331 sq.) - même si, en l'espèce, elle a reconnu la proportionnalité de la mesure contestée, la Constitution n'exigeant pas d'exception générale des journalistes des mesures prises dans le cadre de la lutte contre le terrorisme.

73 BVerfGE 133, 277 pt 116 sq.

74 BVerfGE 141, 220 pt 112.

75 BVerfGE 133, 277 pt $120 s q$.

76 BVerfGE 133, 277 pt 123; 141,220 pt 130 sq.

$77 \quad$ BVerfGE 141, 220 pt. 282 sq., 320.

78 BVerfGE 133, 277 pt 115.

79 BVerfGE 109, $279(377) ; 120,351(369) ; 133,277$ pt. 114.

80 BVerfGE 100, 313 (385 sq.); 109, 279 (379 sq.).

81 Voir par exemple, pour un fichier de terroristes présumés : BVerfGE 133, 277 pts. 224 sq. en ce qui concerne le fichier ; BVerfGE 141, 220 pts. 302 sq. concernant d'autres échanges de données.

82 Cela n'a pas été le cas dans l'affaire BVerfGE 141, 220. pt. 348.

83 BVerfGE 141, 220 pts. $325 s q$.

84 BVerfGE 141, 220 pts. 330 sq.

85 BVerfGE 30, 1 (16) ; 100, 313 (334) ; 113, 348 (363) ; 125, 260 (304 sq.) ; 133, 277 pts. 86 sq. ; 141,220 pt. $80 s q$.

86 BVerfGE 113, $348(392)$; 120, $274(339,344) ; 141,220$ pt. 357.

87 BVerfGE 133, 277 pt 230 ; voir aussi 100, 313 (402) ; 109, 279 (381) ; 110, 33 (76) ; 141, 220 pt. 356.

88 C. GREWE, «Contrôle de constitutionnalité et contrôle de conventionnalité », RFDC 100 (2014), p. 961 (967).

89 V. le rapport de B. W. WEGENER au Congrès de l'Association des professeurs allemands de droit public, «Verfassung in ausgewählten Teilrechtsordnungen - Sicherheitsrecht », Veröffentlichungen der Vereinigung der Deutschen Staatsrechtslehrer 75 (2016), Berlin, de Gruyter, p. 293 sq., et les discussions qui ont suivi.

90 V. cependant J. F. LINDNER, « Die "Karlsruher Republik" - wehrlos in Zeiten des Terrors », Die Öffentliche Verwaltung 2017, p. 90 sq. ; H. A. WOLFF, « Verfassung in ausgewählten Teilrechtsordnungen - Sicherheitsrecht », Deutsches Verwaltungsblatt 2015, p. 1076 sq. 